\title{
BMJ Open Association of pneumococcal conjugate vaccination with rates of ventilation tube insertion in Denmark: population- based register study
}

\author{
Christina Groth, ${ }^{1}$ Reimar W Thomsen, ${ }^{2}$ Therese Ovesen ${ }^{1}$
}

To cite: Groth C,

Thomsen RW, Ovesen T. Association of pneumococcal conjugate vaccination with rates of ventilation tube insertion in Denmark: population-based register study. BMJ Open 2015;5: e007151. doi:10.1136/ bmjopen-2014-007151

- Prepublication history for this paper is available online. To view these files please visit the journal online (http://dx.doi.org/10.1136/ bmjopen-2014-007151).

Received 11 November 2014 Revised 10 March 2015 Accepted 20 March 2015

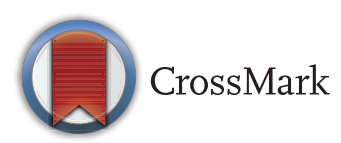

${ }^{1}$ Department of Otorhinolaryngology, Head and Neck Surgery, Aarhus University Hospital, Aarhus C, Denmark

${ }^{2}$ Department of Clinical Epidemiology, Institute of Clinical Medicine, Aarhus University Hospital, Aarhus N, Denmark

Correspondence to Dr Christina Groth; christina.groth@gmail.com

\section{ABSTRACT}

Objective: To examine if the introduction of pneumococcal conjugate vaccine (PCV) in Denmark was associated with a decrease in the rate of ventilation tube (VT) insertions performed by officebased practising ear, nose and throat (ENT) specialists.

Design: Population-based register study based on prospectively collected data.

Setting: Central Denmark Region. Data on VT insertions performed by any office-based practising ENT specialist in the region were collected from the National Health Service Registry.

Participants: All children below the age of 2 years with a first-time VT insertion from 2001 through 2011.

Main outcome measures: Age-stratified and gender-stratified standardised incidence rates of firsttime VT insertion, and incidence rate ratio for PCV period 2008-2011 compared with pre-PCV period 2001-2007.

Results: The annual incidence rate of first-time VT insertion in small children increased steadily from 64/ 1000 person-years in 2001 to $100 / 1000$ person-years in 2011. The incidence rate ratio was $1.27(95 \% \mathrm{Cl}$ 1.24 to 1.30 ) in the PCV period compared with the pre-PCV period.

Conclusions: The introduction of PCV into the Danish childhood immunisation programme in 2007 was not associated with a subsequent decrease in the rate of VT insertions among children below the age of 2 years. Instead, the rate continued to rise, as before the introduction of PCV.

Trial registration number: Danish Data Protection Agency: 2007-58-0010.

\section{INTRODUCTION}

Streptococcus pneumoniae is a major cause of bacterial infection such as meningitis, pneumonia and acute otitis media (AOM) in children and in adults. More than $80 \%$ of children will develop AOM at least once before they reach 3 years of age. ${ }^{1}$ AOM is thus one of the most frequent reasons for children seeing their general practitioner (GP), and $\mathrm{AOM}$ is associated with considerable public
Strengths and limitations of this study

- Population-based design with individual-level data.

- The study includes children from all social layers.

- No recent data on the microbiology of recurrent acute otitis media in Denmark.

- Unknown effect of changes in other risk factors.

health expenses. ${ }^{2}$ S. pneumoniae is a predominant pathogen involved in $\mathrm{AOM}^{3}$ and generally causes more severe AOM than other microorganisms. ${ }^{4}$

Treatment of AOM consists of antibiotic treatment or "watchful waiting'. ${ }^{5}$ However, in many children, the disease turns into recurrent AOM (3 incidents of AOM within 6 months or 5 within a year) and/or otitis media with effusion (OME). Treatment of these complications often includes insertion of a ventilation tube (VT) into the tympanic membrane. VT insertion is the single most frequent surgical procedure performed on children in general anaesthesia in the Western world, and the procedure therefore poses a great socioeconomic burden on most Western societies, ${ }^{6}$ including in Denmark, where the rate of VT insertion is exceptionally high. ${ }^{7}$

The pneumococcal conjugate vaccine (PCV) has been introduced into childhood immunisation programmes worldwide. It is effective in preventing invasive pneumococcal disease (IPD), and probably also pneumonia and AOM, although evidence is less clear for these conditions. ${ }^{8}{ }^{9}$ AOM consultation rates have reportedly decreased by up to $20 \%$ after the introduction of PCV into the childhood immunisation programmes in the USA. ${ }^{10}$ Trials in Finland and the USA suggest that PCV was effective in reducing rates of VT insertion by as much as $39 \% .{ }^{11-14}$ Moreover, postmarketing studies from the 
USA and Australia suggest that the introduction of 7-valent PCV (PCV7) correlates with decreases in VT insertion rates of up to $23 \% .{ }^{15}{ }^{16}$ Similar data from Europe are sparse, and the effect of PCV on pneumococcal disease epidemiology seems to vary greatly between different nations. ${ }^{17}$ Factors potentially contributing to this variation include differences in vaccine schedules, vaccination coverage, antibiotic usage, resistance patterns, and prevalence of bacterial pathogens and pneumococcal serotypes. Surveillance of the potential impact of PCV on AOM and VT insertion rates is therefore needed at a national level.

In Denmark, routine vaccination with PCV7 began in October 2007. The vaccine is given in a $2+1$ dose schedule as part of the Danish childhood immunisation programme. The schedule thus differs from that used in the USA (3+1) and in Australia (3+0). ${ }^{18-20}$

In this study, we examine the association between the introduction of PVC7 and incidence rates of VT insertion in children below the age of 2 years at the population level.

\section{PATIENTS AND METHODS Setting}

The study was conducted in the Central Denmark Region, which has a mixed rural and urban population of 1.27 million inhabitants (23\% of the Danish population). The Danish healthcare service provides universal tax-supported healthcare for all residents, guaranteeing free access to primary medical care and hospitals. GPs act as gatekeepers who refer their clients to treatment at hospitals and to treatment by office-based practising specialists including ear, nose and throat (ENT) specialists. There are approximately 3600 GPs and 1200 practising specialists with agreement under the public healthcare scheme in Denmark. Patients are most often referred to an office-based ENT specialist from their GP, but they may also contact an ENT specialist on their own.

From Statistics Denmark, the central authority on Danish statistics, we obtained data on the number of children living in Central Denmark Region each year, distributed on age group, sex and municipality.

\section{Children with first-time VT insertion}

We identified all children below the age of 2 years treated with VT insertions in the outpatient clinics of practising ENT specialists. The 2-year limit was chosen because recurrent $\mathrm{AOM}$ is more frequent in children below the age of 2 years (peak incidence of AOM is 611 months of age) than in older children in whom OME is predominant. ${ }^{21}$ Nearly all VT insertions in Denmark are performed by office-based practising specialists. There is no waiting time on VT insertion in Denmark, therefore private insurance coverage without reimbursement is practically never used for this procedure. Moreover, VT insertion is reimbursed regardless of whether or not the patient is referred by the GP or the family contacts the ENT specialist without prior referral. All practising ENT specialists report all their patient procedures and services to the Health Care Reimbursement Scheme and are financially reimbursed for their services. The number and type of procedures and services are electronically stored in the Danish National Health Service Registry, together with the date and the patient's unique civil registry number. Information on each patient's birthdate, gender and municipality is also contained in the registry. ${ }^{22}$ The Danish government assigns a unique civil registry number to every citizen at birth. ${ }^{23}$ This allows us to identify all children's first incident VT insertion and ensures that every child appears only once in the calculations. ${ }^{24}$ None of the above facts changed during the calendar period studied. A VT insertion was counted as one episode, regardless of whether insertion was performed unilaterally or bilaterally.

\section{Pneumococcal vaccination and vaccine coverage}

PCV7 was introduced into the Danish childhood immunisation programme in October 2007; and PCV13 was introduced in April 2010. ${ }^{25}$ Since the government recommended GPs to finish use of the remaining stock of PCV7 before starting PVC13 administration, 2010 is considered to be a 'PCV7 year'. Children are vaccinated at 3,5 and 12 months of age. In the introduction period, a catch-up programme was offered to children up to 17 months of age. Children aged 4-11 months were offered three vaccinations with at least 1 month's interval between the first two vaccinations and at least 2 months between the second and the third vaccination. At the time of the third vaccination, the child had to be at least 12 months of age. Children aged 11-17 months were offered two vaccinations with at least 2 months in between. Data on population vaccine coverage were obtained from the Statens Serum Institut (SSI) ${ }^{26}$ which monitors vaccine administration nationally. Vaccine coverage in the Danish Childhood Immunisation Program is estimated using data from the National Health Service Registry and is accessible on an aggregated level from the SSI homepage. ${ }^{26}$ Estimates are available for different basic categories such as gender, birth cohort, municipality and region in Denmark. GPs are obligated to report all vaccinations carried out as a part of the Danish Childhood Immunisation Program to the National Health Service Registry.

\section{Statistical analysis}

Incidence: Annual incidence rates were calculated with the number of people living in the region as of 1 January each year as the denominator. Exact population numbers were retrieved from Statistics Denmark. ${ }^{27}$ The annual incidence rate of VT insertions was computed overall and within age-specific $(0-5,6-11,12-17$ and 18-23 months) and gender-specific strata as the number of first-time VT insertions per 1000 people per year. Furthermore, we computed the annual incidence in municipalities in the eastern versus the western part 
of Central Denmark Region separately. Incidence rate ratios (IRRs) with 95\% CIs were calculated for the PCV period (2008-2011) compared with the pre-PCV period (2001-2007). Incidence rates and rate ratios were age and gender standardised to the population in 2001. The 95\% CIs were considered statistically significant.

\section{RESULTS}

We identified 27837 children below the age of 2 years with a first-time VT insertion from 2001 through 2011. The mean age at the first VT insertion was 14.8 months and $59 \%$ of the children were male.

\section{Vaccine coverage}

PCV coverage in the Central Denmark Region was estimated at $86-93 \%$ for the first PCV for the birth cohorts from 2007 through 2011. The coverage was estimated at $82-93 \%$ for the second PCV dose and at $81-92 \%$ for the third PCV dose in the birth cohorts from 2007 through 2011. The coverage for children in the catch-up programme was $51-71 \%$ depending on age and PCV dose number.

\section{Incidence of first VT insertion}

Between 2001 and 2011, the incidence rate of first-time VT insertion in children below the age of 2 years steadily increased from 64/1000 person years in 2001 to 100 / 1000 person years in 2011. Small temporary decreases appeared in 2005, 2008 and 2011. The overall IRR was 1.27 (95\% CI 1.24 to 1.30 ) when the PCV period was compared with the pre-PCV period (figure 1). Age-specific rate ratios are listed in table 1.

The VT insertion rate clearly depended on both age group and gender. During the entire period, males had a substantially higher incidence rate than females; in

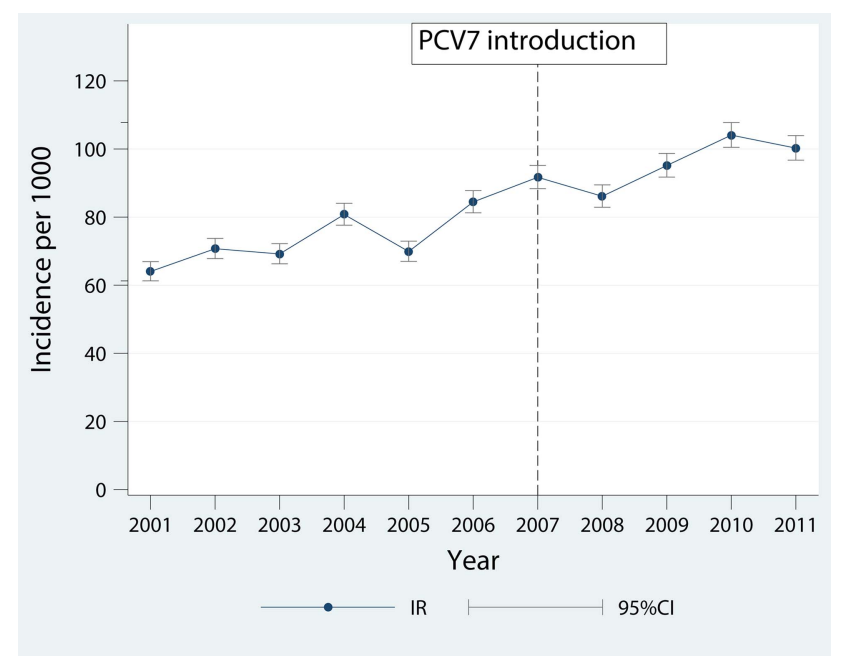

Figure 1 Standardised overall IR for first-time VT insertion in Central Denmark Region before and after the introduction of PCV in October 2007 (IR, incidence rate; PCV7, 7-valent pneumococcal conjugate vaccine; VT, ventilation tube).
2011, the rate for males was $111 / 1000$ person years, while the corresponding number for females was 81/ 1000 person years. Moreover, the incidence rate was clearly higher in children aged 12-17 months than in the other age groups. Nonetheless, the increasing trend of VT insertion rates was seen for both genders and for all age groups. Furthermore, the trend was similar in the western and the eastern part of the Central Denmark Region, although the overall insertion rate tended to be highest in the western part (figures 2-4).

\section{DISCUSSION}

Our population-based study shows that the introduction of PCV into the Danish childhood immunisation programme in 2007 was not associated with a subsequent decrease in the rates of VT insertions in the Central Denmark Region. In contrast, the rate continued to rise. The small decreases in the rates seen in 2005, 2008 and 2011 could be due to natural fluctuations in the incidence of otitis media and subsequent VT insertions, for instance related to viral epidemics.

The strengths of this study include its populationbased design with individual-level data, which yields a large number of cases and enables us to focus on incident first-time VT insertion episodes only. The Danish healthcare system provides free healthcare for all residents. Our study therefore includes children from all social layers and not children only from specific clinics or health insurance systems.

The possible limitations of the study include potential coding errors in the healthcare registry. Nonetheless, owing to direct economic incentives, coding of procedures in the Danish National Health Service Registry is considered highly valid. ${ }^{28}$ The children vaccinated against pneumococci in our population are not necessarily the same as those later having or avoiding VT insertion. We are therefore faced with an inborn risk of ecological fallacy when inferring any association. On the other hand, the high PCV vaccination rate in the Central Denmark Region, where around $90 \%$ of the children are covered, is likely to induce herd immunity, and one may argue that the ecological design is, in fact, a strength. Changes over time in other AOM and VT risk factors might have influenced the observed rates of VT insertions. Important risk factors for recurrent AOM and therefore likely risk factors also for VT insertion include parental smoking, day care attendance and lack of breast feeding. ${ }^{29}$ The prevalence of tobacco smokers in Denmark decreased during the study period, ${ }^{30}$ whereas the percentage of children attending day care has risen over the past decade. ${ }^{31}$ Data on changes over time in the prevalence of breast feeding in Denmark are, unfortunately, lacking. Lastly and importantly, we have no recent data on the microbiology of recurrent $\mathrm{AOM}$ in Denmark.

In Denmark, the main criteria for VT insertion are recurrent AOM or OME for more than 3 months. We 
Table 1 Mean IR of first VT insertion per 1000 person years in the PCV period (2008-2011) and the pre-PCV period (20012007), standardised to the 2001 population

\begin{tabular}{lcrr}
\hline Age group (months) & IR, PCV period $(\mathbf{n})$ & IR, pre-PCV period $(\mathbf{n})$ & IRR $(\mathbf{9 5 \%} \mathbf{C l )}$ \\
\hline All & $96.3(11637)$ & $76.0(16200)$ & $1.27(1.24$ to 1.30$)$ \\
$0-5$ & $2.6(78)$ & $1.7(89)$ & $1.56(1.15$ to 2.12$)$ \\
$6-11$ & $109.0(3242)$ & $84.7(4488)$ & $1.29(1.23$ to 1.35$)$ \\
$12-17$ & $182.4(5530)$ & $140.3(7507)$ & $1.30(1.26$ to 1.35$)$ \\
$18-23$ & $91.9(2787)$ & $76.9(4116)$ & $1.20(1.14$ to 1.25$)$ \\
\hline
\end{tabular}

IRR with 95\% Cls.

IR, incidence rate; IRR, incidence rate ratio; PVC, pneumococcal conjugate vaccine; VT, ventilation tube.

had no data on the indication for the VT procedures performed. Increasing rates of OME with subsequent VT insertion could have caused the rise in VT incidence rates and could have masked any effect of the PCV on the incidence of AOM. Only a few studies have examined the medical indications underlying VT insertions. Two studies found that recurrent AOM was the indication for VT insertion in $20 \%{ }^{24}$ and $40 \%{ }^{25}$ of cases. Another study ${ }^{15}$ found that $93 \%$ of children at 1 year of age and $95 \%$ of children at 2 years of age had developed frequent episodes of otitis media at the time of VT insertion. However, these studies were conducted in the USA and Australia, where much fewer VTs are inserted among children than is the case in Denmark.

Furthermore, it is well known that AOM and OME represent different stages within a continuum of otitis media. Most children suffer from a mixture of recurrent $\mathrm{AOM}$ and OME, which makes it difficult to exclusively ascribe VT insertion to either of the two indications.

We had no data on possible changes in the use of antibiotics specifically for AOM treatment in our study period, which might have affected the rate of VT insertions. In recent years, there has been a general focus on reducing antibiotic use in Denmark, including antibiotic treatment for AOM, emphasising 'watchful waiting' as an option in uncomplicated AOM. ${ }^{5}$ Less antibiotic use for AOM could have led to increased choice of VT insertion. Nonetheless, between 2003 and 2012, the total consumption of antimicrobial agents in humans in Denmark increased by 3.44 defined daily doses per 1,000 inhabitants per day (DID) or $23 \%,{ }^{32}$ and we have no data to indicate reduced antibiotic use for AOM in our study period.

No previous study has evaluated the association of PCV with otitis-related outcomes in Denmark. However, our findings of a continually increasing rate of VT insertions are consistent with a recent study by Djurhuus et $a l^{7}$ who also found increasing rates of VT insertions in Denmark. In contrast, studies from other countries, including the $\mathrm{USA}^{15}$ and Australia, ${ }^{33}$ have reported decreases in VT insertion rates following the introduction of PCV. While the rate of IPD in Denmark fell substantially in the vaccinated age-groups following the PCV7 introduction, ${ }^{34}$ incidence rates of hospitalised pneumonia did not decrease, but rose between 2007 and 2011 in Denmark. ${ }^{35}$ Thus, while PCV prevents IPD,
Figure 2 Standardised IRs for first-time VT insertion in Central Denmark Region before and after the introduction of PCV in October 2007, stratified by geography (IR, incidence rate; PCV7, 7-valent pneumococcal conjugate vaccine; VT, ventilation tube).

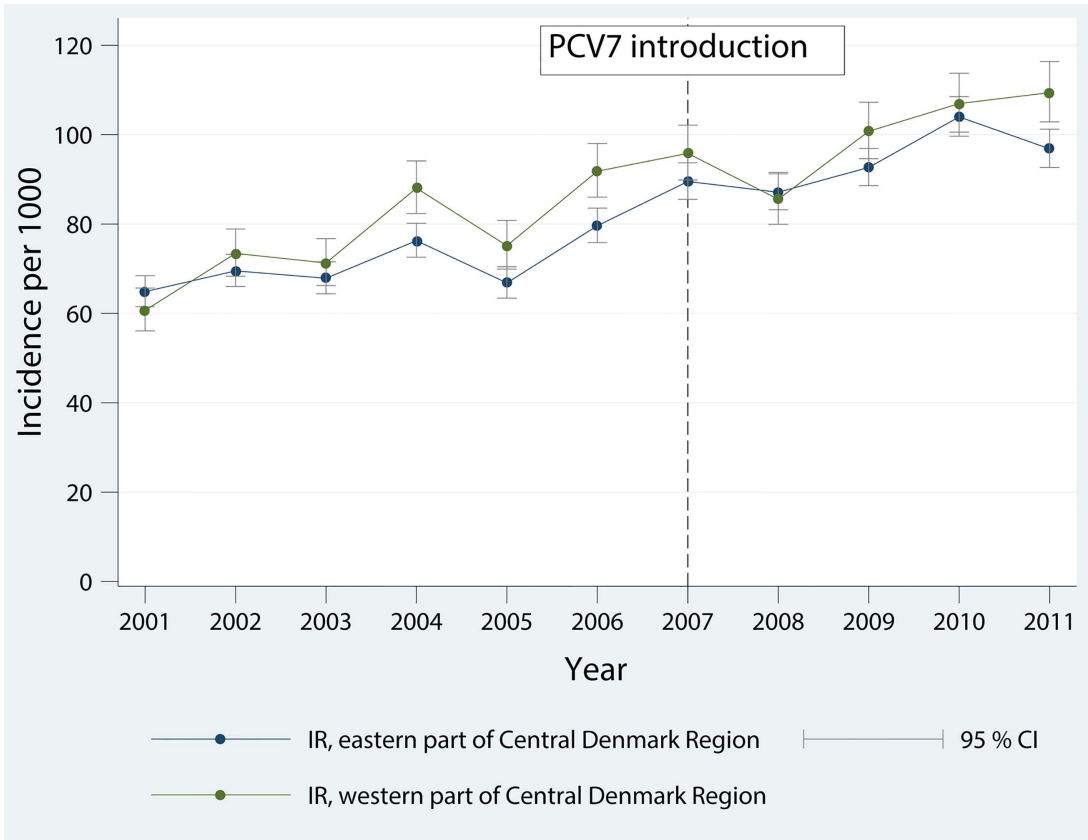


Figure 3 Standardised IRs for first-time VT insertion in Central Denmark Region before and after the introduction of PCV in October 2007, stratified by gender (IR, incidence rate; PCV7, 7-valent pneumococcal conjugate vaccine; VT, ventilation tube).

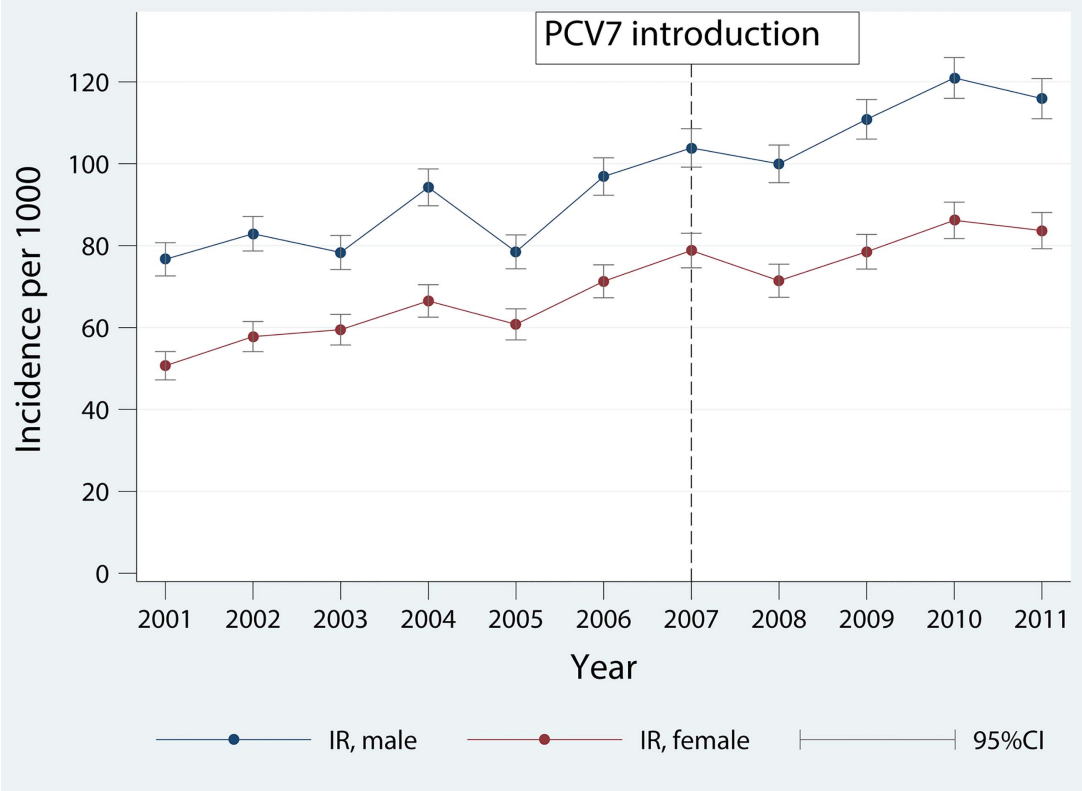

it may have less effect on non-IPD. In Finland, pneumococcal vaccination induced a serum IgG immune response from 7 months of age, ${ }^{36}$ but an immune response in saliva IgA could not be detected until 16 months of age. This could explain why the vaccine protects children below the age of 2 years from IPD, but apparently not from mucosal disease such as AOM and pneumonia. Also, previously mentioned variations in vaccine schedules could result in different outcomes in countries like Denmark, the USA and Australia.

An additional explanation for lack of effect could be the occurrence of serotype replacement. Several studies show an increase in non-vaccine types (NVT) after the introduction of PCV7. Ingels $e t a l^{44}$ reported a slight, but significant, increase in NVT IPD in Denmark in the PCV period for all age groups. For children below the age of 2 years, the IRR was only 1.18 (95\% CI 0.91 to 1.53$)$. However, the distribution of serotypes and serotype replacement could be different for non-IPD such as AOM.

The observed increase in VT insertion rates started long before the implementation of PCV, and there could be numerous reasons for this trend. A growing number of children may be diagnosed with recurrent $\mathrm{AOM}$ either because the incidence of $\mathrm{AOM}$ is rising or because of increased use of medical care. Thus, data from The Danish National Health Service Registry and the National Board of Health show that the annual number of GP consultations and the medical expenses per Danish citizen have risen continuously since 2001. ${ }^{37}$ However, the increase in the overall share of medical
Figure 4 Standardised IRs for first-time VT insertion in Central Denmark Region before and after the introduction of PCV in October 2007, stratified by age group (IR, incidence rate; PCV7, 7-valent pneumococcal conjugate vaccine; VT, ventilation tube).

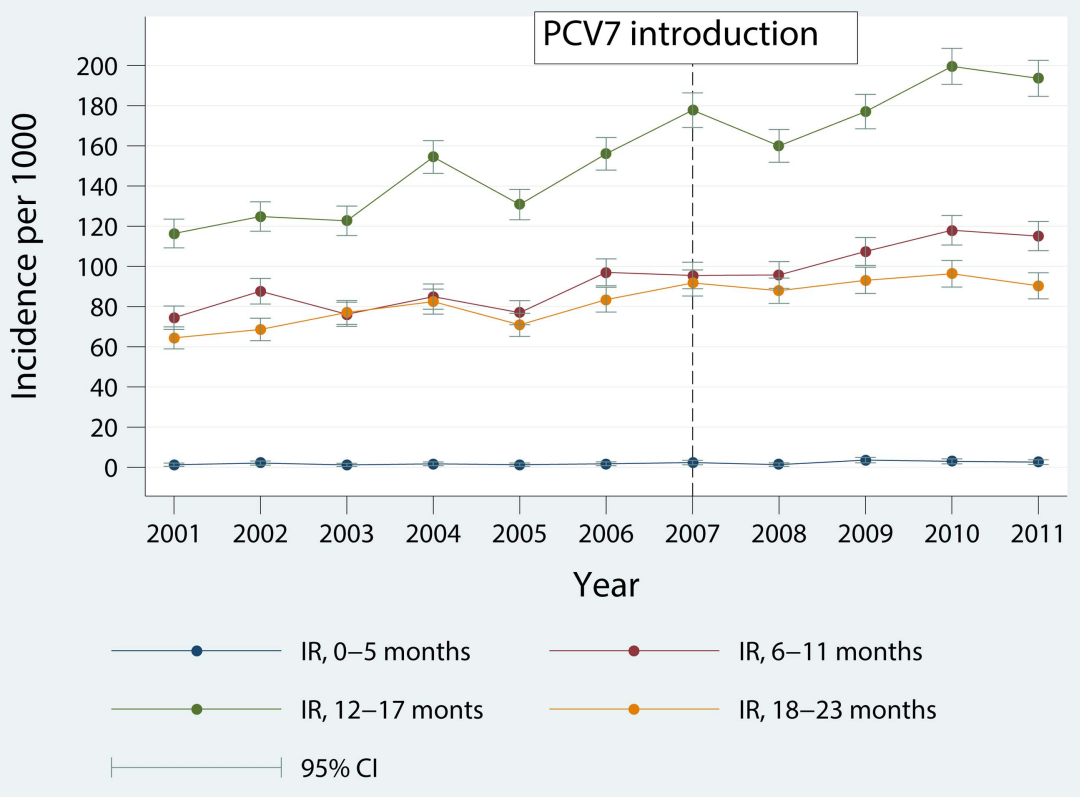


expenses of Denmark's gross domestic product from $8.6 \%$ in 2001 to $9.7 \%$ in 2007 does not compare with the large increase in VT insertions observed in this period.

A study from New York ${ }^{6}$ showed that VT insertion was an overused procedure and that $69.7 \%$ of VT insertions were performed without the child fulfilling the criteria for the procedure. If this holds true for Denmark as well, the increasing rate could reflect growing overuse of the procedure. While a Cochrane review ${ }^{38}$ from 2008 showed that VT insertion rests on a rather limited scientific base and only has a small effect in reducing episodes of recurrent AOM, a quality assessment survey from Denmark ${ }^{39}$ in 2009 showed that parents with children undergoing VT insertion were very pleased with the procedure. Thus, the survey found that $96 \%$ of parents were satisfied with the treatment. Parents reported improved night sleep (of the child) in $78 \%$, less ear pain in $85 \%$ and a better quality of life of the family in $66 \%$ of the cases. In the same survey, $15 \%$ of the ENT specialists felt that the parents had forced them to perform the procedure. Denmark has the highest employment proportion of women in the world, and both parents have fulltime employment in most Danish families. A sick child is therefore a large burden on the family because one of the parents has to stay home from work to take care of the child. As a result, frustrated parents might pressure their GP and practising specialist to take action and will not be satisfied with a "watchful waiting' approach. Increased parental pressure during the study period could have led to an increased rate of VT insertions. No data exist on changes in parental attitude in Denmark during the period studied.

Finally, changes over time in official criteria and guidelines for VT insertion could have affected the rate of procedures. The last official national treatment guideline for VT insertions from 1987 states that indications include recurrent $\mathrm{AOM}$ and $\mathrm{OME}$ for more than 3 months. ${ }^{40}$ However, during the last decade, there has been an ongoing debate within the Danish health community in favour of more restricted indications for VT insertions. Recent unofficial clinical recommendations-the latest from $2014^{41}$-suggest OME for more than 6 months as indication for possible VT insertion. A Danish assessment ${ }^{42}$ of the previously discussed Cochrane review concluded that VT insertions are not without complications; and in light of the relative small effect, each procedure should be carefully considered. It seems that official steps are being taken to implement more restrictive criteria, which, however, is not consistent with the rise in the number of procedures performed reported in the present study. A new official national guideline for VT insertion is expected to be published in 2015 .

\section{CONCLUSION}

In conclusion, we found that the introduction of PCV into the Danish childhood immunisation programme did not correlate with a subsequent decrease in the rates of VT insertions for children below the age of 2 years. Instead, the rate in Denmark has continued to rise, as before the introduction of PCV.

Contributors CG, TO and RWT conceived the study idea and design. CG collected and carried out analysis of the data, directed by TO and RWT. All authors participated in the discussion and interpretation of the results. CG wrote the initial draft of the manuscript. All authors revised the manuscript and approved the final version. TO is the guarantor.

Funding The study was supported by the Oticon Foundation (grant number 11-3239), Ørelæge Hans Skoubys og Hustru Emma Skoubys Fond and Speciallæge i Øre-næse-halssygdomme Poul Traun-Pedersens mindelegat.

Competing interests None declared.

Ethics approval The study was approved by the Danish Data Protection Agency (J.no. 2007-58-0010).

Provenance and peer review Not commissioned; externally peer reviewed.

Data sharing statement No additional data are available.

Open Access This is an Open Access article distributed in accordance with the Creative Commons Attribution Non Commercial (CC BY-NC 4.0) license, which permits others to distribute, remix, adapt, build upon this work noncommercially, and license their derivative works on different terms, provided the original work is properly cited and the use is non-commercial. See: http:// creativecommons.org/licenses/by-nc/4.0/

\section{REFERENCES}

1. Vergison A, Dagan R, Arguedas A, et al. Otitis media and its consequences: beyond the earache. Lancet Infect Dis 2010;10:195-203

2. Alsarraf R, Jung CJ, Perkins J, et al. Measuring the indirect and direct costs of acute otitis media. Arch Otolaryngol Head Neck Surg 1999;125:12-18.

3. Block SL, Hedrick J, Harrison CJ, et al. Community-wide vaccination with the heptavalent pneumococcal conjugate significantly alters the microbiology of acute otitis media. Pediatr Infect Dis $J$ 2004;23:829-33.

4. Cunningham M, Guardiani E, Kim HJ, et al. Otitis media. Future Microbiol 2012;7:733-5.

5. American Academy of Pediatrics Subcommittee on Management of Acute Otitis Media. Diagnosis and management of acute otitis media. Pediatrics 2004;113:1451-65.

6. Keyhani S, Kleinman LC, Rothschild M, et al. Overuse of tympanostomy tubes in New York metropolitan area: evidence from five hospital cohort. BMJ 2008;337:a1607.

7. Djurhuus BD, Skytthe A, Christensen $\mathrm{K}$, et al. Increasing rate of middle ear ventilation tube insertion in children in Denmark. Int $J$ Pediatr Otorhinolaryngol 2014;78:1541-4.

8. Fitzwater SP, Chandran A, Santosham M, et al. The worldwide impact of the seven-valent pneumococcal conjugate vaccine. Pediatr Infect Dis J 2012;31:501-8.

9. Black S, Shinefield H, Fireman B, et al. Efficacy, safety and immunogenicity of heptavalent pneumococcal conjugate vaccine in children. Northern California Kaiser Permanente Vaccine Study Center Group. Pediatr Infect Dis J 2000;19:187-95.

10. Grijalva CG, Poehling KA, Nuorti JP, et al. National impact of universal childhood immunization with pneumococcal conjugate vaccine on outpatient medical care visits in the United States. Pediatrics 2006;118:865-73.

11. Palmu AA, Verho J, Jokinen J, et al. The seven-valent pneumococcal conjugate vaccine reduces tympanostomy tube placement in children. Pediatr Infect Dis J 2004;23:732-8.

12. Sarasoja I, Jokinen J, Lahdenkari M, et al. Long-term effect of pneumococcal conjugate vaccines on tympanostomy tube placements. Pediatr Infect Dis J 2013;32:517-20.

13. Palmu A. Effectiveness of the 10-valent pneumococcal conjugate vaccine against tympanostomy tube placements in a clusterrandomized trial. 7th Extraordinary International Symposium on Recent Advances in Otitis Media, Stockholm, Sweden 12-16 June, 2013.

14. Fireman B, Black SB, Shinefield HR, et al. Impact of the pneumococcal conjugate vaccine on otitis media. Pediatr Infect Dis J 2003;22:10-16. 
15. Poehling KA, Szilagyi PG, Grijalva CG, et al. Reduction of frequent otitis media and pressure-equalizing tube insertions in children after introduction of pneumococcal conjugate vaccine. Pediatrics 2007;119:707-15.

16. Jardine A, Menzies RI, Deeks SL, et al. The impact of pneumococcal conjugate vaccine on rates of myringotomy with ventilation tube insertion in Australia. Pediatr Infect Dis $J$ 2009;28:761-5

17. Rozenbaum MH, Boersma C, Postma MJ, et al. Observed differences in invasive pneumococcal disease epidemiology after routine infant vaccination. Expert Rev Vaccines 2011;10:187-99.

18. Børnevaccinationsprogrammet. http://www.ssi.dk/Vaccination/ Boernevaccination/Boernevaccinationsprogrammet.aspx (accessed 8 March 2015).

19. Immunization schedules. http://www.cdc.gov/vaccines/schedules/ hcp/imz/child-adolescent.html (accessed 8 March 2015).

20. National Immunisation Program Schedule. http://www.immunise. health.gov.au/internet/immunise/publishing.nsf/Content/nips-ctn (accessed 8 March 2015).

21. Zielhuis $\mathrm{GA}$, Rach $\mathrm{GH}$, van den Bosch $\mathrm{A}$, et al. The prevalence of otitis media with effusion: a critical review of the literature. Clin Otolaryngol Allied Sci 1990;15:283-8.

22. Andersen JS, Olivarius Nde F, Krasnik A. The Danish National Health Service Register. Scand J Public Health 2011;39:34-7.

23. Pedersen CB. The Danish Civil Registration System. Scand J Public Health 2011;39:22-5.

24. Frank L. Epidemiology. When an entire country is a cohort. Science 2000;287:2398-9.

25. PCV-tilslutning og invasiv pneumokoksygdom. 2010. http://www.ssi. dk/Aktuelt/Nyhedsbreve/EPI-NYT/2011/Uge\%2019\%20-\%202011. aspx.

26. SSI overvågning. http://www.ssi.dk/Smitteberedskab/Sygdomsover vaagning.aspx.

27. http://www.statistikbanken.dk

28. Nickelsen TN. Data validity and coverage in the Danish National Health Registry. A literature review. Ugeskr Laeger 2001;164:33-7.

29. Rovers MM, de Kok IM, Schilder AG. Risk factors for otitis media: an international perspective. Int $J$ Pediatr Otorhinolaryngol 2006;70:1251-6.
30. Voksnes rygevaner. http://www.cancer.dk/forebyg/roegfri/fakta +rygning/Voksnes+rygevaner/.

31. Børnepasning i Danmark. http://www.statistikbanken.dk/statbank5a/ default.asp?w=1440 (accessed 21 November 2012).

32. DANMAP. Use of antimicrobial agents and occurrence of antimicrobial resistance in bacteria from food animals, food and humans in Denmark. ISSN 1600-2032. 2012. http://www.danmap.org.

33. Spilsbury K, Kadhim AL, Semmens JB, et al. Decreasing rates of middle ear surgery in Western Australian children. Arch Otolaryngol Head Neck Surg 2006;132:1216-20.

34. Ingels $\mathrm{H}$, Rasmussen J, Andersen $\mathrm{PH}$, et al. Impact of pneumococcal vaccination in Denmark during the first 3 years after PCV introduction in the childhood immunization programme. Vaccine 2012;30:3944-50.

35. Sogaard M, Nielsen RB, Schonheyder HC, et al. Nationwide trends in pneumonia hospitalization rates and mortality, Denmark 1997-2011. Respir Med 2014;108:1214-22.

36. Nurkka A, Ahman H, Korkeila M, et al. Serum and salivary anti-capsular antibodies in infants and children immunized with the heptavalent pneumococcal conjugate vaccine. Pediatr Infect Dis $J$ 2001;20:25-33.

37. Det danske sundhedsvæsen i internationalt perspektiv. http://www. sst.dk/publ/Publ2010/DOKU/OECD/DKsundhedsv internationaltpersp.pdf.

38. McDonald S, Langton Hewer CD, Nunez DA. Grommets (ventilation tubes) for recurrent acute otitis media in children. Cochrane Database Syst Rev 2008;(4):CD004741.

39. Johansen EC, Svendstrup B, Schonsted-Madsen U, et al. Parents satisfied with the grommet treatment in ear, nose and throat practice. Ugeskr Laeger 2010;172:2530-4.

40. [No authors listed]. Danish approach to the treatment of secretory otitis media. Report of The Danish Consensus Conference. Copenhagen, 1987. Ann Otol Rhinol Laryngol Suppl 1990;146:1-28.

41. Mellemørebetændelse. http://laegehaandbogen.dk/ore-nese-hals/ tilstande-og-sygdomme/mellemore/mellemorebetendelse-seros3531.html

42. Lous J, Trankjaer CR, Thomsen J. Grommets (ventilation tubes) for recurrent acute otitis media in children-assessment of a Cochrane review. Ugeskr Laeger 2010;172:2526-30. 